\title{
Mechanical cavopulmonary assist maintains pulmonary and cerebral blood flow in a piglet model of a bidirectional cavopulmonary shunt with high pulmonary vascular resistance
}

\author{
Osami Honjo, MD, PhD, ${ }^{\text {a }}$ Sandra L. Merklinger, RN, PhD, ${ }^{\text {a } J o h n ~ B . ~ P o e, ~ M S c, ~}{ }^{\text {a }}$ Anne-Marie Guerguerian, MD, \\ Abdullah A. Alghamdi, MD, ${ }^{a}$ Setsuo Takatani, PhD, DMed, ${ }^{\mathrm{c}}$ and Glen S. Van Arsdell, $\mathrm{MD}^{\mathrm{a}}$
}

Objectives: We tested mechanical cavopulmonary blood flow assist by incorporating a novel miniature centrifugal pump into a $1 \frac{1}{2} 2$-ventricle type cavopulmonary connection in neonatal pigs.

\begin{abstract}
Methods: Nine 3-week-old piglets (mean body weight, $10.2 \mathrm{~kg}$ ) were used: mechanical cavopulmonary assist $(n=6)$ and controls $(n=3)$. A bidirectional cavopulmonary connection between the superior vena cava and the main pulmonary artery was created. The superior vena cava and pulmonary artery were also connected by cannulas with an interposed centrifugal pump. The cavoarterial mechanical cavopulmonary assist was performed at pump speeds of 1500, 2000, 2500, and $3000 \mathrm{rpm}$. Retrograde superior vena caval flow was limited by a band on the superior vena cava. A bidirectional cavopulmonary connection was created in the control animals, which then had a pure 1 1/2-ventricle repair physiology without mechanical support. Hemodynamics, blood gas, and cerebral blood flow measured by ultrasound were analyzed. Catheter-based dilatation of the surgically created superior vena cava obstruction was tested.
\end{abstract}

Results: Incremental increases in pump speed augmented bidirectional cavopulmonary shunt blood flow $(P=.03)$ and diminished superior vena caval pressure $(P=.03)$, thereby improving cerebral perfusion pressure. Pump flow of $3000 \mathrm{rpm}$ was equivalent to baseline superior vena caval flow (before caval flow, $392 \pm 48 \mathrm{~mL} / \mathrm{min}$ vs MCPA, $371 \pm 120 \mathrm{~mL} / \mathrm{min}$; mean $\pm \mathrm{SD} ; P=$ not significant). The mechanical cavopulmonary assist group had higher Doppler velocities of the middle cerebral artery and higher transcerebral oxygen difference $(P<.05)$ than controls. Balloon dilatation of the superior vena cava band was successful.

Conclusions: Mechanical cavopulmonary assist maintained bidirectional cavopulmonary shunt flow, thereby sustaining primary bilateral cavopulmonary shunt physiology in a neonatal pig model of high pulmonary vascular resistance. The mechanical cavopulmonary assist maintained cerebral blood flow and metabolism with an adequate transcerebral pressure gradient.

Overall outcomes of patients with hypoplastic left heart syndrome have improved over the past 2 decades with advances in surgical and perioperative therapeutic strategies. ${ }^{1,2}$ However, all forms of current stage 1 surgical palliation have an inherently unstable "in-parallel" circulation. We have hypothesized that this is a cause of early and interim morbidity and mortality. ${ }^{3-5}$ "In-series" physiology is established at the time of the stage II bidirectional cavopulmonary shunt (BCPS). Morbidity and mortality are diminished with stage II physiology. One might therefore also hypothesize that sur-

\footnotetext{
From The Labatt Family Heart Centre ${ }^{\mathrm{a}}$ and Pediatric Critical Care Unit, ${ }^{\mathrm{b}}$ Hospital for Sick Children and the University of Toronto, Toronto, Ontario, Canada, and the ${ }^{\mathrm{c}}$ Department of Artificial Organs, Institute of Biomaterial and Bioengineering, Tokyo Medical Dental University, Tokya, Japan.

Read at the Eighty-eighth Annual Meeting of The American Association for Thoracic Surgery, San Diego, Calif, May 10-14, 2008.

Received for publication May 3, 2008; revisions received Aug 3, 2008; accepted for publication Sept 16, 2008.

Address for reprints: Glen S. Van Arsdell, MD, Division of Cardiovascular Surgery, Hospital for Sick Children, 555 University Ave, Toronto, Ontario, Canada, M5G 1X8 (E-mail: glen.vanarsdell@sickkids.ca).

J Thorac Cardiovasc Surg 2009;137:355-61

$0022-5223 / \$ 36.00$

Copyright (c) 2009 by The American Association for Thoracic Surgery

doi:10.1016/j.jtcvs.2008.09.036
}

gical outcomes might be improved by primary application of an in-series palliation with a BCPS in the neonatal or early infantile period. The first attempt of a primary BCPS in a 5-week-old infant with hypoplastic left heart syndrome, by Dr William I. Norwood in 1977, resulted in early death with progressive desaturation. ${ }^{6}$ Subsequent series of early BCPS application in early infancy also showed high mortality mainly related to severe hypoxemia and pulmonary artery (PA) thrombosis. ${ }^{7,8}$

Immaturity of the peripheral pulmonary vasculature and high pulmonary vascular resistance (PVR) preclude successful application of a primary BCPS in neonates and small infants. Peripheral pulmonary vasculature develops in the months after birth so that PVR becomes low enough to create a cavopulmonary connection usually at 5 to 6 months of age. We hypothesized that establishment of a primary BCPS circulation in neonates might be feasible if the lungs are assisted by a mechanical pump, thereby overcoming the problems related to pulmonary vascular immaturity. This proof-of-concept study was designed to test a "mechanical cavopulmonary assist" (MCPA) system as a means to improve forward BCPS flow in the presence of high $\mathrm{PVR}$, much as is seen in the human neonate. We examined 


$$
\begin{aligned}
& \text { Abbreviations and Acronyms } \\
& \begin{aligned}
\text { BCPS } & =\text { bidirectional cavopulmonary shunt } \\
\text { CPB } & \text { cardiopulmonary bypass } \\
\text { CPP } & =\text { cerebral perfusion pressure } \\
\text { JB } & \text { jugular bulb } \\
\text { MCA } & =\text { middle cerebral artery } \\
\text { MCPA } & \text { mechanical cavopulmonary assist } \\
\text { NS } & =\text { not significant } \\
\text { PA } & \text { pulmonary artery } \\
\text { PVR } & =\text { pulmonary vascular resistance } \\
\text { RA } & =\text { right atrium } \\
\text { SVC } & \text { superior vena cava }
\end{aligned}
\end{aligned}
$$

assisted pulmonary blood flow rates and cerebral blood flow. As a second component, we sought to demonstrate the possibility of a catheter-based completion to unassisted BCPS circulation.

\section{MATERIALS AND METHODS}

All experimental protocols were approved by the Animal Care Committee at the Hospital for Sick Children. Nine 3-week-old pigs (mean body weight, $10.2 \mathrm{~kg}$ ) were divided into two groups: the MCPA group $(\mathrm{n}=6)$ and the control group $(\mathrm{n}=3)$. The animals were premedicated with ketamine $(20 \mathrm{mg} / \mathrm{kg})$, atropine $(0.02 \mathrm{mg} / \mathrm{kg})$, and acepromazine $(2 \mathrm{mg} / \mathrm{kg})$ before endotrachial intubation. Mechanical ventilation (Air-Shields Inc, Hatboro, Pa) was maintained with a tidal volume of 10 to $15 \mathrm{~mL} / \mathrm{kg}$ and $50 \%$ oxygen and $2 \%$ isoflurane. Minor ventilator adjustments were made to maintain normal blood gas values (partial pressure of carbon dioxide, 35-45 mm Hg; pH, 7.35-7.44). Temperature was maintained at $37^{\circ} \mathrm{C}$ with a thermal heating pad placed under the animals. The left femoral artery and vein, the right internal jugular vein, and the jugular bulb (JB) were cannulated for pressure monitoring and blood sampling. The heart was exposed through a median sternotomy and the pericardium suspended. The superior vena cava (SVC) and main PA were completely mobilized. The bilateral azygos veins were ligated and divided to mobilize the SVC. Direct left atrial and distal PA pressures were monitored via $3 \mathrm{~F}$ catheters. SVC and aortic blood flow was measured by an ultrasonic flow probe (Transonic Systems, Ithaca, NY) before surgery.

After systemic heparinization (400 U/kg), a temporary shunt was created between the SVC and right atrium (RA) with $12 \mathrm{~F}$ right-angled venous cannulas. The SVC was transected at its junction to the RA, and the RA was oversewn. The main PA was partially clamped, and a transverse incision was made on the anterior wall of the main PA approximately $1 \mathrm{~cm}$ above the pulmonary ring. The SVC was anastomosed to the main PA in an end-to-side fashion with 5-0 or 6-0 polypropylene sutures (Ethicon, Inc, Somerville, NJ) (Figure 1, A). The SVC was anastomosed to the main $\mathrm{PA}$, as opposed to the right $\mathrm{PA}$, because in pigs the right $\mathrm{PA}$ is posterior, making the anastomosis in that location technically impractical. The final anatomy was that of a $1 \frac{1}{2}$-ventricle repair. The temporary SVC-to-RA shunt was eliminated after completion of the anastomosis. A $10 \mathrm{~F}$ arterial cannula was inserted into the main PA distal to the BCPS anastomosis. The $5 \mathrm{~mL}-$ prime miniature centrifugal pump ${ }^{9}$ was then connected via the cannulas placed in the SVC and the distal main PA (Figure 1, B), and MCPA was initiated and carried out to a maximum of 2 hours. Retrograde SVC flow caused by the pump and right ventricular pulsatility was limited by a band placed on the SVC between the cannula and anastomotic site. A tourniquet was initially used and later was replaced by a metal wire band once hemodynamic stability was achieved (Figure $1, C$ ). Pump initiation without the SVC band led to hemodynamic instability related to circular blood flow. The band tightness was adjusted to the level that yielded systemic hemodynamic stability, indicating that circular blood flow was now not clinically significant. A BCPS was created in the control animals under a temporary shunt as described above. After the anastomosis, the cannulas placed in the SVC and RA were removed, making a pure $1 \frac{1}{2} 2$-ventricle repair physiology without mechanical support.

The hematocrit value was maintained at a baseline level with supplemental blood from a donor pig if necessary. In the MCPA group, blood gas and hemodynamics were analyzed every 10 minutes at pump speeds of 1500 , 2000, 2500, and $3000 \mathrm{rpm}$. The blood gas was analyzed 10 minutes after BCPS creation in the control group. Centrifugal pump flow was continuously monitored by an ultrasonic flow probe (Transonic Systems, Ithaca, NY). Finally, an 8F Mullins dilatation catheter (NuMED, Inc, Hopkinton, $\mathrm{NY}$ ) was inserted via the right internal jugular vein to the SVC across the band, and the surgically created obstruction was dilated when the pump flow was terminated and the SVC cannula was removed.

\section{Evaluation of Cerebral Blood Flow and Metabolism}

Cerebral perfusion pressure (CPP) was calculated using the difference between mean arterial and JB pressures. Transcerebral oxygen and lactate differences were calculated from arterial and JB partial oxygen pressures and lactate levels. Peak systolic, diastolic, and mean velocities and velocity time integral of the right middle cerebral artery (MCA) were measured by transcranial Doppler ultrasound (Vivid 7; GE Vingmed, Horten, Norway) using a 5- to 8-MHz probe to estimate cerebral blood flow volume ${ }^{10}$ at baseline and 10 minutes after maximal MCPA of $3000 \mathrm{rpm}$ in the MCPA group. Doppler ultrasound was performed at baseline and 10 minutes after BCPS creation in the control group.

\section{Statistical Methods}

Data are presented as means \pm standard deviations. Comparisons between preoperative and postoperative values were performed by the paired $t$ test. Comparisons of the values between the MCPA and control groups were performed by Wilcoxon rank-sum test. Changes in hemodynamic and metabolic parameters were determined by 1-way analysis of variance, followed by a post hoc test using the Bonferroni multiple comparisons test.

\section{RESULTS}

\section{The Effect of Pump Speed on Hemodynamics and Metabolism}

Incremental increases in the centrifugal pump speed augmented BCPS blood flow $(P=.03)$ and diminished SVC pressure $(P=.03)$, thereby improving CPP $(P=.03)$ (Table 1). The final pump flow of $3000 \mathrm{rpm}$ was equivalent to the SVC flow before the procedure (pre-SVC flow, $392 \pm 48$ $\mathrm{mL} / \mathrm{min}$, vs MCPA, $371 \pm 120 \mathrm{~mL} / \mathrm{min}$; mean $\pm \mathrm{SD} ; P=$ not significant $[\mathrm{NS}])$. All MCPA animals were hemodynamically stable, well ventilated, and oxygenated until being humanely killed 2 hours after pump initiation.

\section{Effects of Mechanical Lung Assist on 11/2-Ventricle Repair Physiology}

Hemodynamics, metabolism, and cerebral blood flow patterns were compared between the MCPA group and the control animals. No statistical differences in baseline parameters were noted between the groups (Table 2). Comparisons in hemodynamics and metabolism between the MCPA group with a pump speed of $3000 \mathrm{rpm}$ and the control group are 


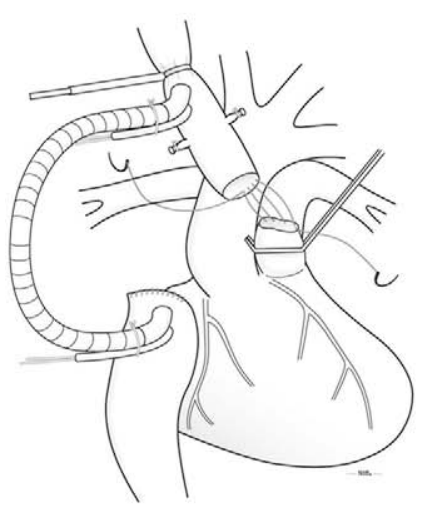

A
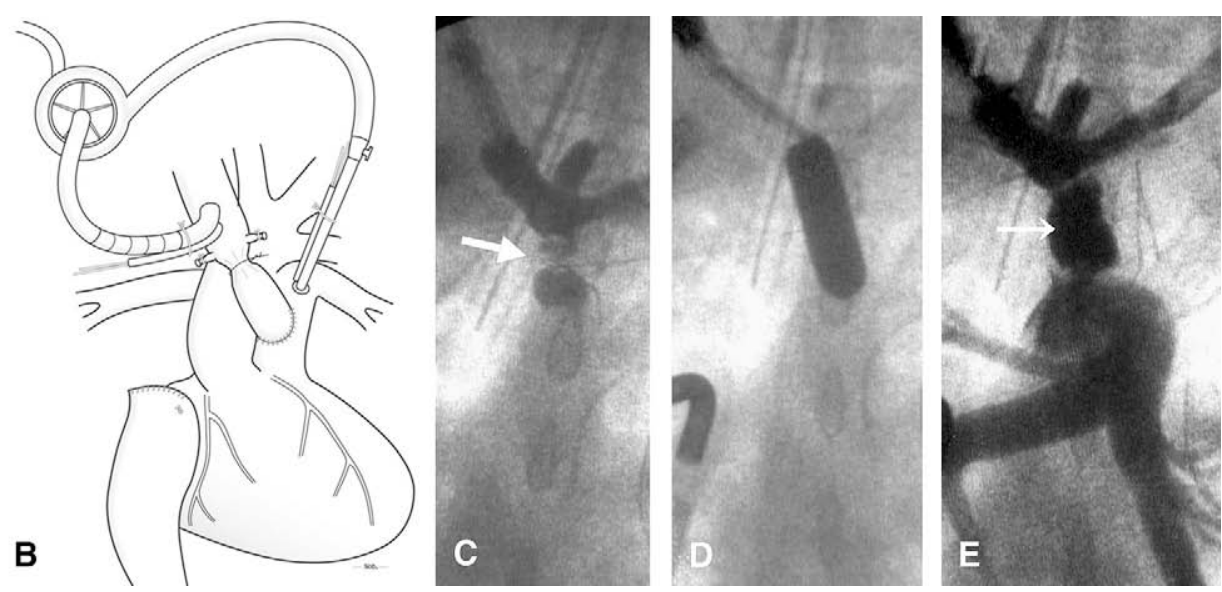

FIGURE 1. A, Creation of BCPS under a temporary shunt. The main PA was partially clamped and the SVC was anastomosed to the PA in an end-to-side fashion. B, Miniature centrifugal pump assist after BCPS completion. A band was placed around the SVC distal to the cannula to prevent retrograde flow. The temporary SVC-RA shunt was removed. C, Angiogram of the SVC after BCPS anastomosis. An SVC band (arrow) allows minimal retrograde blood flow. D, Balloon dilatation of the banded SVC. E, Restoration of BCPS circulation. No stenosis remained at the banded region (arrow). There is evidence of some stenosis at the anastomotic site, which is technical in nature.

shown in Table 3. Mean blood pressure was lower in the control group but was not statistically significant. Control animals had higher SVC $(P=.0014)$ and JB pressures $(P=.0008)$. In contrast, the MCPA group maintained a higher $\mathrm{PaO}_{2}(P=.04)$ and mixed venous saturation $(P<.0001)$ whereas lactate in this group remained lower $(P=.012)$ compared with control animals. Pump flow in the MCPA group was higher than the SVC flow in the control group $(P=.0054)$.

\section{Cerebral Blood Flow and Metabolism}

No statistical differences in the baseline MCA flow velocities and velocity time integral, and transcerebral oxygen and lactate levels were noted between the two groups $(P=\mathrm{NS})$. Transcranial Doppler ultrasound measurement showed persistence of baseline MCA velocities and velocity time integral in the MCPA group in contrast to a decrease in these values in control animals (Figure 2). Reduction in the MCA flow velocity in the control group was largest in diastole.

CPP was equivalent to baseline in the MCPA group versus a reduction in CPP in the control animals $(P<.001)$ (Table 4). Similarly, transcerebral oxygen difference was comparable with baseline in the MCPA group but lower in the control animals $(P<.0001)$. No difference in the transcerebral lactate levels was detected among the groups.

Clinical demonstration of a possible conversion to unassisted SVC flow via a percutaneous method was shown with successful balloon dilatation of the SVC band (Figure 1, $C, D$, and $E$ ).

All 6 animals in the MCPA group maintained hemodynamic stability throughout the study period, whereas all 3 control animals died within 20 minutes of BCPS creation. Two of the control animals had progressive hypotension and subsequent cardiac arrest and the third pig had refractory

TABLE 1. Hemodynamic and blood gas parameters in MCPA animals at 4 different pump speeds

\begin{tabular}{|c|c|c|c|c|c|}
\hline & $1500 \mathrm{rpm}$ & $2000 \mathrm{rpm}$ & $2500 \mathrm{rpm}$ & $3000 \mathrm{rpm}$ & $P$ value \\
\hline BCPS flow $(\mathrm{mL} / \mathrm{min})$ & $147 \pm 63$ & $233 \pm 80$ & $325 \pm 100$ & $370 \pm 120^{*}$ & $.03 *$ \\
\hline Heart rate (beats/min) & $131 \pm 38$ & $126 \pm 33$ & $127 \pm 32$ & $117 \pm 27$ & NS \\
\hline MAP $(\mathrm{mm} \mathrm{Hg})$ & $40.0 \pm 4.6$ & $40.3 \pm 4.8$ & $40.2 \pm 4.5$ & $41 \pm 4.1$ & NS \\
\hline SVCp (mm Hg) & $14.0 \pm 1.8$ & $11.5 \pm 3.1$ & $7.3 \pm 6.0^{*}$ & $5.5 \pm 3.7^{*}$ & $.03^{*}$ \\
\hline $\mathrm{JBP}(\mathrm{mm} \mathrm{Hg})$ & $13.5 \pm 2.3$ & $11.5 \pm 2.3$ & $10 \pm 2.4$ & $9.25 \pm 2.2$ & NS \\
\hline $\mathrm{CPP}(\mathrm{mm} \mathrm{Hg})$ & $26.5 \pm 4.5$ & $27.0 \pm 5.2$ & $30.2 \pm 5.6$ & $38.3 \pm 12.2^{*}$ & $.03^{*}$ \\
\hline mPAP $(\mathrm{mm} \mathrm{Hg})$ & $21.3 \pm 8.5$ & $21.0 \pm 7.6$ & $19.3 \pm 6.2$ & $18.5 \pm 4.6$ & NS \\
\hline LAP $(\mathrm{mm} \mathrm{Hg})$ & $6.0 \pm 8.0$ & $6.2 \pm 7.8$ & $6.2 \pm 7.8$ & $6.7 \pm 7.5$ & NS \\
\hline $\mathrm{pH}$ & $7.38 \pm 0.03$ & $7.39 \pm 0.03$ & $7.39 \pm 0.04$ & $7.41 \pm 0.06$ & NS \\
\hline $\mathrm{PaCO}_{2}(\mathrm{~mm} \mathrm{Hg})$ & $39.8 \pm 0.8$ & $39.9 \pm 1.7$ & $38.8 \pm 2.1$ & $36.7 \pm 3.1$ & NS \\
\hline $\mathrm{PaO}_{2}(\mathrm{~mm} \mathrm{Hg})$ & $357.3 \pm 31.5$ & $356.7 \pm 36.6$ & $351.3 \pm 20.9$ & $336.0 \pm 33.8$ & NS \\
\hline Lactate $(\mathrm{mmol} / \mathrm{L})$ & $4.1 \pm 1.7$ & $3.9 \pm 1.1$ & $3.5 \pm 0.9$ & $3.1 \pm 0.8$ & NS \\
\hline
\end{tabular}

Values expressed as means \pm SD. $M C P A$, Mechanical cavopulmonary assist; $B C P S$, bidirectional cavopulmonary shunt; $M A P$, mean arterial pressure; $S V C p$, superior vena cava pressure; $J B P$, jugular bulb pressure; $C P P$, cerebral perfusion pressure, $m P A P$, mean pulmonary artery pressure; $L A P$, left atrial pressure; $N S$, not significant. $* P<.05$ compared with $1500 \mathrm{rpm}$. 
TABLE 2. Baseline parameters between MCPA and control group

\begin{tabular}{|c|c|c|c|}
\hline & MCPA group $(n=6)$ & Control $(n=3)$ & $P$ value \\
\hline Body weight (kg) & $10.1 \pm 1.1$ & $10.3 \pm 1.5$ & NS \\
\hline Heart rate (beats/min) & $125 \pm 13$ & $130 \pm 15$ & NS \\
\hline MAP (mm Hg) & $46.3 \pm 2.6$ & $55.3 \pm 1.8$ & NS \\
\hline SVCp (mm Hg) & $7.2 \pm 3.2$ & $5.6 \pm 1.6$ & NS \\
\hline $\mathrm{JBP}(\mathrm{mm} \mathrm{Hg})$ & $9.2 \pm 1.3$ & $7.0 \pm 2.6$ & NS \\
\hline CPP (mm Hg) & $37.5 \pm 3.9$ & $45.2 \pm 3.1$ & NS \\
\hline mPAP (mm Hg) & $16.2 \pm 1.6$ & $17.7 \pm 1.8$ & NS \\
\hline LAP (mm Hg) & $3.7 \pm 0.3$ & $4.3 \pm 0.3$ & NS \\
\hline PVR (Wood units) & $11.9 \pm 3.7$ & $13.1 \pm 3.4$ & NS \\
\hline $\mathrm{pH}$ & $7.43 \pm 0.02$ & $7.46 \pm 0.02$ & NS \\
\hline $\mathrm{PaCO}_{2}(\mathrm{~mm} \mathrm{Hg})$ & $39.9 \pm 1.2$ & $38.1 \pm 3.1$ & NS \\
\hline $\mathrm{PaO}_{2}(\mathrm{~mm} \mathrm{Hg})$ & $260 \pm 23$ & $301 \pm 30$ & NS \\
\hline Lactate $(\mathrm{mmol} / \mathrm{L})$ & $1.7 \pm 0.4$ & $1.8 \pm 0.3$ & NS \\
\hline SVC flow $(\mathrm{mL} / \mathrm{min})$ & $392 \pm 48$ & $506 \pm 56$ & NS \\
\hline Aortic flow & $882 \pm 69$ & $943 \pm 20$ & NS \\
\hline Hemoglobin (g/dL) & $8.5 \pm 0.12$ & $8.5 \pm 0.21$ & NS \\
\hline
\end{tabular}

Values expressed as means $\pm \mathrm{SD}$. MCPA, Mechanical cavopulmonary assist; $M A P$, mean arterial pressure; $S V C p$, superior vena cava pressure; $J B P$, jugular bulb pressure; $C P P$, cerebral perfusion pressure, $m P A P$, mean pulmonary artery pressure; $L A P$, left atrial pressure; $P V R$, peripheral vascular resistance; SVC, superior vena cava; $N S$, not significant.

ventricular fibrillation as a result of hypotension and desaturation.

\section{DISCUSSION}

Creation of a primary BCPS in patients with hypoplastic left heart syndrome is intriguing because it would provide a more stable in-series circulation. Those who have attempted a BCPS in very young infants have reported severe hypoxemia, PA thrombosis, and high mortality and morbidity owing to pulmonary vascular immaturity. ${ }^{7,8}$ We hypothesized that the previously noted problems could be overcome with mechanical assistance. The present study demonstrates that mechanical support with a miniature centrifugal pump can normalize SVC flow in the presence of elevated PA pressures caused by the pulsatility of a $1 \frac{1}{2} 2$-ventricle configuration and neonatal pig physiology. Intrinsic instability issues with pigs and cardiopulmonary bypass (CPB) limited our ability to duplicate a neonatal BCPS. This model contains the essential components of relatively high PVR and neonatal physiology but allows the procedure to be performed without CPB. With MCPA, normal SVC pressures, ventilation, and oxygenation were achieved. Hemodynamic and metabolic stability were maintained. Finally, MCPA also sustained low SVC and JB pressures and normal arterial pressure, allowing for preservation of normal cerebral blood flow. The control animals allow demonstration that the pump provided effective support to overcome high PVR. The physiology of the control animals, however, is different from that of a neonatal single ventricle with a BCPS.

The implications of this proof-of-concept study are that the need for an in-parallel neonatal circulation might be
TABLE 3. Hemodynamic and metabolic comparisons between MCPA (pump speed, $3000 \mathrm{rpm}$ ) and control group

\begin{tabular}{lccc}
\hline & $\begin{array}{c}\text { MCPA (3000 rpm) } \\
(\mathbf{n}=\mathbf{6})\end{array}$ & Control $(\mathbf{n}=\mathbf{3})$ & $\boldsymbol{P}$ value \\
\hline Heart rate (beats/min) & $128 \pm 25$ & $152 \pm 18$ & $\mathrm{NS}$ \\
$\mathrm{MAP}(\mathrm{mm} \mathrm{Hg})$ & $47 \pm 11$ & $34 \pm 9$ & $\mathrm{NS}$ \\
$\mathrm{SVCp}(\mathrm{mm} \mathrm{Hg})$ & $6.6 \pm 3.6$ & $20.3 \pm 4.1^{*}$ & $0.0014^{*}$ \\
$\mathrm{JBP}(\mathrm{mm} \mathrm{Hg})$ & $9.3 \pm 1.7$ & $17.3 \pm 2.5^{*}$ & $0.0008^{*}$ \\
$\mathrm{CVP}(\mathrm{mm} \mathrm{Hg})$ & $5.8 \pm 2.6$ & $6.1 \pm 4$ & $\mathrm{NS}$ \\
$\mathrm{mPAP}(\mathrm{mm} \mathrm{Hg})$ & $16.2 \pm 1.6$ & $17.7 \pm 1.8$ & $\mathrm{NS}$ \\
$\mathrm{LAP}(\mathrm{mm} \mathrm{Hg})$ & $3.7 \pm 0.3$ & $4.3 \pm 0.3$ & $\mathrm{NS}$ \\
$\mathrm{pH}$ & $7.38 \pm 0.07$ & $7.35 \pm 0.05$ & $\mathrm{NS}$ \\
$\mathrm{PaCO}_{2}(\mathrm{~mm} \mathrm{Hg})$ & $40.3 \pm 6.3$ & $38.6 \pm 1.5$ & $\mathrm{NS}$ \\
$\mathrm{PaO}_{2}(\mathrm{~mm} \mathrm{Hg})$ & $324 \pm 35$ & $209 \pm 136^{*}$ & $0.04^{*}$ \\
$\mathrm{SaO}_{2}(\%)$ & $99.5 \pm 0.5$ & $96 \pm 5.2$ & $\mathrm{NS}$ \\
$\mathrm{SvO}_{2}(\%)$ & $83.8 \pm 2.5$ & $41.9 \pm 9.4^{*}$ & $<0.0001^{*}$ \\
$\mathrm{Lactate}^{*}(\mathrm{mmol} / \mathrm{L})$ & $3.1 \pm 0.6$ & $6.0 \pm 1.9^{*}$ & $0.012^{*}$ \\
$\mathrm{BCPS} \mathrm{flow}(\mathrm{mL} / \mathrm{min})$ & $427 \pm 155$ & $58 \pm 22^{*}$ & $0.0054^{*}$ \\
\hline
\end{tabular}

Values expressed as means \pm SD. $M C P A$, Mechanical cavopulmonary assist; $M A P$, mean arterial pressure; $S V C p$, superior vena cava pressure; $J B P$, jugular bulb pressure; $C V P$, central venous pressure, $m P A P$, mean pulmonary artery pressure; $L A P$, left atrial pressure; $B C P S$, bidirectional cavopulmonary shunt. ${ }^{*} P<.05$ compared with MCPA group.

eliminated. A lung circulatory assist device would need to be used for the duration required to achieve adequate lung maturation-potentially weeks to several months. The concept of multiple months' mechanical circulatory assist for young infants has already been established for failing hearts, ${ }^{11,12}$ indicating that the possibility of an appropriately designed mechanical circulatory assist of BCPS blood flow to the lungs might be achieved. Once adequate lung maturation has been achieved, explantation of the system could occur along with percutaneous relief of the surgically created SVC-PA junction obstruction. Further studies focusing on thrombotic problems, chronic lung maturation, and pump reliability using a long-term model remain to be performed.

Impairment of cerebral perfusion and metabolism caused by relative venous hypertension is one of the major concerns associated with BCPS creation in infants with high PVR and PA pressure. In the control group, BCPS creation resulted in severe impairment of the MCA blood flow patterns, especially in diastole. This phenomenon was previously documented in a clinical attempt of BCPS creation using a simple clamping technique. In this strategy, diastolic flow disappeared as the brain lost an effective transcerebral pressure gradient as a result of high SVC pressures. ${ }^{13}$ Of interest, brain metabolism in the control group appeared to be maintained on the basis of transcerebral oxygen and lactate differences despite the significant drop in cerebral blood flow 10 minutes after BCPS creation, presumably owing to metabolic reserve of the brain for acute hypoperfusion. On the other hand, MCPA effectively maintained normal cerebral perfusion and metabolism by decompressing SVC pressure and by keeping an effective transcerebral pressure 

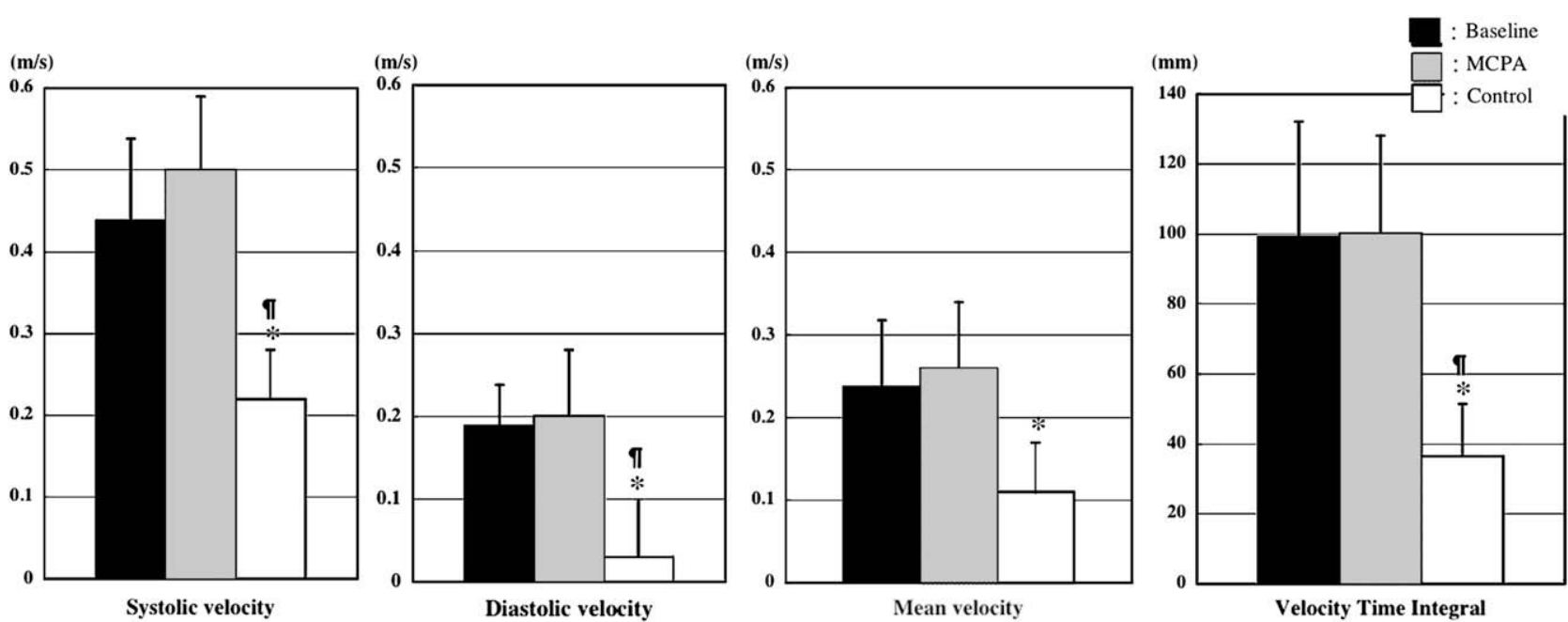

FIGURE 2. Transcranial Doppler ultrasound flow velocities in the middle cerebral arteries of the MCPA group and the control group compared with the baseline value. Values expressed as means $\pm \mathrm{SD}$. $*<.01$ compared with the MCPA group; $\mathbb{\uparrow}<.01$ compared with the baseline value.

gradient. This is an important piece of information. MCPA for a BCPS maintains normal cerebral blood flow, thus allowing a further exploration of multiple months' mechanical assist while waiting for adequate lung maturation.

An animal model of 11/2-ventricle repair physiology rather than a single ventricle physiology was used in this study. We previously created a single left ventricle model by creating a large intra-atrial communication, resecting tricuspid valve leaflets, and occluding the main PA. A BCPS served as the pulmonary blood flow source. This original study essentially showed that the MCPA augmented survival and improved oxygenation and ventilation. ${ }^{14}$ However, the major limitations of the study were the significant deterioration of systemic metabolism characterized by high lactate, low $\mathrm{pH}$, and requirements for substantial volume infusion even with the pump assist. Neonatal pigs are generally vulnerable to $\mathrm{CPB}$ exposure, which can produce severe pulmonary hypertension associated with capillary leak and hemodynamic and metabolic deterioration. ${ }^{15}$ These issues limited our ability to investigate delicate changes in cerebral perfusion and metabolism. Therefore, we moved to a model that allowed us to study components of the physiology. In this instance it was cerebral metabolism and perfusion. In the previous study it was the component of ventilation and oxygenation. Because

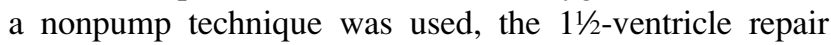
physiology model allowed accurate investigation of the impact of BCPS and MCPA on pulmonary and cerebral per- fusion. Despite the avoidance of $\mathrm{CPB}$, the failure rate before reaching data acquisition or completing the surgical component of the procedure was as high as $50 \%$. This was primarily due to the intolerance of the neonatal pig to anesthesia and/or cardiac manipulation. Nevertheless, the utility of this model was sufficient to test the ability to assist blood flow in the upper venous system to the lungs and to evaluate the effects of the MCPA system on pulmonary and cerebral perfusion in the presence of a very high PVR.

We used a novel miniature centrifugal pump with a priming volume of $5 \mathrm{~mL}$ and a flow range of 0.1 to $4.0 \mathrm{~L} / \mathrm{min} .{ }^{9,16}$ The flexible pump flow range and small surface area are advantageous when considering mechanical assist in growing small infants. A miniaturized axial flow pump, similar to the Impella system, ${ }^{17,18}$ would be an alternative option to support BCPS physiology. A small axial flow pump might be directly inserted into the SVC, which would be ideal to achieve nonoperative explantation. At present, a small axial flow pump that could be inserted into the SVC in small infants does not exist. There remains some room to investigate which type of pump is best for incorporation into an MCPA system. Further animal studies along with the development of small mechanical pumps are warranted.

The strategy of primary in-series palliation with MCPA in the neonatal period might be enhanced if the normal maturation process of the lungs could be accelerated. Exposure to the mechanical pump and the related risks, such as

TABLE 4. Comparisons of cerebral blood flow and metabolism between baseline and after BCPS creation with or without MCPA

\begin{tabular}{lcccc}
\hline & Baseline & MCPA & Control & $\boldsymbol{P}$ value \\
\hline $\mathrm{CPP}(\mathrm{mm} \mathrm{Hg})$ & $45.6 \pm 8.3$ & $38.3 \pm 12.3$ & $20.1 \pm 8.1^{* \dagger}$ & $<.001$ \\
$\mathrm{O}_{2} \mathrm{~A}-\mathrm{V}$ difference $(\mathrm{mm} \mathrm{Hg})$ & $234.5 \pm 17.7$ & $244.6 \pm 20$ & $81.6 \pm 91.8^{* \dagger}$ & $<.0001$ \\
Lactate A-V difference $(\mathrm{mmol} / \mathrm{L})$ & $0.27 \pm 0.72$ & $0.13 \pm 0.1$ & $0.2 \pm 0.17$ & $\mathrm{NS}$ \\
\hline$B C P S$, Bit & &
\end{tabular}

$B C P S$, Bidirectional cavopulmonary shunt; $M C P A$, mechanical cavopulmonary assist; $C P P$, cerebral perfusion pressure; $A-V$, atriovenous. ${ }^{*} P<.05$ compared with baseline. $\dagger P<.05$ compared with MCPA group. 
thromboembolism, hemolysis, and mechanical failure, could be minimized if the maturation period were shortened. We have reported that both unfractionated and low molecular weight heparin accelerate pulmonary vascular maturation in neonatal rabbits by $250 \%$ and that this was characterized by significantly lower PA pressure at 2 weeks of age. ${ }^{19}$ Further development of this "chemo-mechanical lung assist" strategy might facilitate a paradigm shift in the surgical treatment of hypoplastic left heart syndrome. Many issues remain to be explored before this strategy can be applied clinically.

\section{Study Limitations}

There are certain limitations in this study. First, this is not a true cyanotic single ventricle model, but rather a high PVR cavopulmonary shunt model. Therefore, this study provided little data regarding the effects of MCPA on systemic hemodynamic, metabolism, and oxygenation and ventilation in a functional single ventricle physiology. Second, the short duration of the study provided little data regarding the possibility of long-term cavopulmonary assist using this type of system. A long-term study will be required to focus on, among other factors, anticoagulation, thrombosis, and pump sustainability. Third, the banded SVC, which was successfully dilated with a balloon catheter, may not be so simply dilated after long-term mechanical assist. Finally, the band placed on the SVC prevented circular flow from the PA to the SVC in the MCPA group. It also prevented retrograde flow in the SVC from right ventricular ejection. Because this is a $1 \frac{1}{2}$-ventricle model, the control animals would have had retrograde flow in the SVC during right ventricular ejection. As such, the control animals' cerebral physiology would be different from a single ventricle model of a high PVR cavopulmonary connection.

\section{CONCLUSIONS}

MCPA maintained BCPS flow, thereby sustaining the $1 \frac{1}{2}$-ventricle repair physiology with reasonable systemic hemodynamics and metabolism in neonatal pigs. This approach also maintained cerebral blood flow and metabolism by efficiently decompressing SVC pressure and maintaining an adequate transcerebral pressure gradient. This study conceptually raises the possibility of a primary BCPS creation in neonates with the aid of MCPA followed by catheter-based explantation of the pump system.

We thank Lisa Davey, Marvin Estrada, Lauren McKeeman, Stephen Y. Ta-min, and Satoko Uematsu for their technical support. We also thank Nobuko Yamamoto for her excellent illustrations.

\section{References}

1. Azakie T, Merklinger SL, McCrindle BW, Van Arsdell GS, Lee KJ, Benson LN, et al. Evolving strategies and improving outcomes of the modified Norwood procedure: a 10-year single-institution experience. Ann Thorac Surg. 2001;72: 1349-53.
2. Mahle WT, Spray TL, Wernovsky G, Gaynor JW, Clark BJ 3rd. Survival after reconstructive surgery for hypoplastic left heart syndrome: a 15-year experience from a single institution. Circulation. 2000;102(19 Suppl 3): III136-41.

3. Stasik CN, Goldberg CS, Bove EL, Devaney MD, Ohye RG. Current outcomes and risk factors for the Norwood procedure. J Thorac Cardiovasc Surg. 2006; 131:412-7.

4. Mahle WT, Spray TL, Gaynor JW, Clark BJ. Unexpected death after reconstructive surgery for hypoplastic left heart syndrome. Ann Thorac Surg. 2001; 71:61-5.

5. Bartram U, Grunenfelder J, Van Praaph R. Causes of death after the modified Norwood procedure: a study of 122 postmortem cases. Ann Thorac Surg. 1997;64: 1795-802.

6. Norwood WI, Kirklin JK, Sanders SP. Hypoplastic left heart syndrome: experience with palliative surgery. Am J Cardiol. 1980;45:87-91.

7. Reddy VM, Liddicoat JR, Hanley FL. Primary bidirectional superior cavopulmonary shunt in infants between 1 and 4 months of age. Ann Thorac Surg. 1995;59: 1120-5.

8. Bradley SM, Mosca RS, Hennein HA, Crowley DC, Kulik TJ, Bove EL. Bidirectional superior cavopulmonary connection in young infants. Circulation. 1996; 94(9 Suppl):II5-11.

9. Takatani S, Hoshi H, Tajima K, Ohuchi K, Nakamura M, Asama J, et al. Feasibility of a miniature centrifugal rotary blood pump for low-flow circulation in children and infants. ASAIO J. 2005;51:557-62.

10. Evans N, Kluckow M, Simmons M, Osborn D. Which to measure, systemic or organ blood flow? Middle cerebral artery and superior vena cava flow in very preterm infants. Arch Dis Child Fetal Neonatal. 2002;87:F181-4.

11. Chang AC, McKenzie ED. Mechanical cardiopulmonary support in children and young adults: extracorporeal membrane oxygenation, ventricular assist devices, and long-term support devices. Pediatr Cardiol. 2005;26:2-28.

12. Stiller B, Weng Y, Hubler M, Lemmer J, Nagdyman N, Redlin M, et al. Pneumatic pulsatile ventricular assist devices in children under 1 year of age. Eur Cardiothorac Surg. 2005;28:234-9.

13. Rodriguez RA, Cornel G, Austin EH, Auden SM, Weerasena NA. Brain function monitoring during bidirectional Glenn procedures. J Thorac Cardiovasc Surg. 2000;119:617-9.

14. Merklinger SL, Honjo O, Al-Radi OO, Poe J, Wang JZ, Van Arsdell GS. An animal model of mechanical lung assist for primary in-series palliation of hypoplastic left heart syndrome. Circulation. 2006;114:II544-5.

15. Carteauz JP, Roux S, Siaphy M, Schjoth B, Dolofon P, Bechamps Y, et al. Acute pulmonary hypertension after cardiopulmonary bypass in pig: the role of endogenous endothelin. Eur J Cardiothorac Surg. 1999;15:346-52.

16. Kido K, Hoshi H, Watanabe N, Kataoka H, Ohuchi K, Asama J, et al. Computational fluid dynamics analysis of the pediatric tiny centrifugal blood pump (TinyPump). Artif Organs. 2006;30:392-9.

17. Patel S, Allaire PE, Wood HG, Song X, Throckmorton AL, Untaroiu A, et al. Axial flow blood pumps. ASAIO J. 2003;49:255-64

18. Hanke T, Notzold A, Olschewski M, Siegenthaler MP, Brehm K, Strecker T, et al. The Impella Recover microaxial left ventricular assist device reduced mortality for postcardiotomy failure: a three-center experience. $J$ Thorac Cardiovasc Surg. 2004;127:812-22.

19. O’Blenes SB, Merklinger SL, Jegatheeswaran A, Campbell A, Rabinovitch M, Rebeyka I, et al. Low molecular weight heparin and unfractionated heparin are both effective at accelerating pulmonary vascular maturation in neonatal rabbits. Circulation. 1003;108(Suppl 1):II161-6.

\section{Discussion}

Dr William Gaynor (Philadelphia, $\mathrm{Pa}$ ). Why did you use the $1 \frac{1}{2}$-ventricle model? Why not ligate the main PA? In your no-assist group the PA is not banded, thus the right ventricle is able to eject and you are going to have a high SVC pressure. Basically, by banding the assist group you are just looking at a pump decompression of the SVC. It does not seem like a fair comparison of the two groups. Why not clamp the main PA and look at a pure superior cavopulmonary connection model rather than a $1 \frac{1}{2} 2$-ventricle model?

Dr Honjo. Thank you for your question. There are a couple of reasons why we used this model. The advantage of this 
model is that we can actually separate the cerebral circulation from the systemic circulation, looking at the effect of the pump system on the cerebral circulation and the metabolism. However, the disadvantage, as you mentioned, is that this is not a single ventricle model, meaning this is not a cyanotic model. We cannot compare the systemic oxygenation or ventilation. We have actually done the single ventricle model in which we created the single left ventricle under CPB with a cavopulmonary shunt as the primary blood source. That essentially showed that mechanical support extended the survival and also improved carbon dioxide exchange and oxygenation. However, because we had to use CPB, which deteriorates the systemic metabolism, that is not a good model to compare the cerebral perfusion in a fine way. That is why in this particular study we used this model.
Dr Y. Joseph Woo (Philadelphia, $\mathrm{Pa}$ ). With the way you displayed your model, depending on the flow rate that you establish within your pump, you could establish some degree of a closed loop circulation. How did you titrate how tightly to band the SVC to prevent such a closed loop circulation?

Dr Honjo. That is a very good question. This SVC band is very tight, because we do not want retrograde flow getting into the pump system, which makes a noneffective circulation. We do not want to occlude the SVC because we would eventually use it for a cavopulmonary shunt. However, this SVC band that we use is very tight. This is one of the disadvantages of using this pump system. Actually, if you use a different pump system, like a small axial pump, you can insert it into the SVC, and we do not need such a band. It might be beneficial, but we do not have a pump small enough to be inserted into the SVC. 